\title{
Synthesis of novel pyridine containing azetidinone derivatives as a potential anti tubercular activity
}

\author{
N. Pramod ${ }^{1, *}$, B. Mayuri ${ }^{2}$ \\ ${ }^{1} \mathrm{HOD},{ }^{2}$ Assistant Professor, Dept. of Pharmaceutical, Annamachrya College of Pharmacy, Rajampet, Kadapa, Andhra Pradesh,
} India

*Corresponding Author:

Email: pramodnayanapalli@gmail.com

\begin{abstract}
Six novel pyridine containing azetidinones Azt 1-6 have been synthesized from Schiff base by treating Pyridine-4-carbaldehyde with Substituted aromatic amines each were taken equimolar concentration in presence of Glacial acetic acid as a catalyst using Grindstone technique. Further Schiff base add benzene, triethylamine and chloro acetyl chloride undergoes condensation followed by elimination reaction result into novel pyridine containing azetidinone derivatives. The newly synthesized derivatives were characterized by using spectroscopic methods (IR, ${ }^{1} \mathrm{H}-\mathrm{NMR}, \mathrm{MASS}$ ) and screened for selected anti-tubercular. In vitro antitubercular activity was performed using Micro plate Alamar Blue Assay (MABA) method. Among synthesized compounds Azt5 and azt6 showed more potent Anti TB activity than standards.
\end{abstract}

Keywords: Pyridine-4-carbaldehyde, Schiff base, Azetidinones, anti-tubercular activity.

\section{Introduction}

Pyridine is a basic heterocyclic organic compound with the chemical formula $\mathrm{C}_{5} \mathrm{H}_{5} \mathrm{~N}$. It is structurally related to benzene, with one methine group $(=\mathrm{CH}-)$ replaced by a nitrogen atom. The pyridine ring occurs in many important compounds, including azines and vitamins niacin and pyridoxine.

Pyridine is used as

a precursor to agrochemicals and pharmaceuticals and is also an important solvent and reagent. ${ }^{1}$ Some chemical compounds, although not synthesized from pyridine, contain its ring structure. They include B vitamins niacin and pyridoxine, the antituberculosis drug isoniazid, nicotine and other nitrogencontaining plant products. ${ }^{2}$ Pyridine is a colorless liquid that boils at $115.2{ }^{\circ} \mathrm{C}$ and freezes at $-41.6{ }^{\circ} \mathrm{C}$. Its density, $0.9819 \mathrm{~g} / \mathrm{cm}^{3}$, is close to that of water, and its refractive index is 1.5093 at a wavelength of $589 \mathrm{~nm}$ and a temperature of $20^{\circ} \mathrm{C} .{ }^{3}$

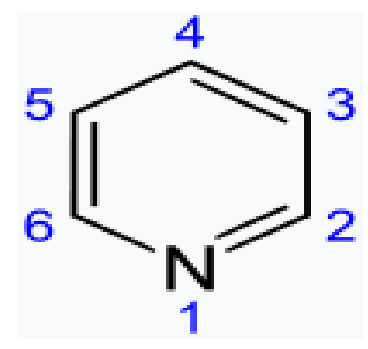

Pyridine is used as a precursor to agrochemicals and pharmaceuticals and is also an important solvent and reagent. Pyridine is added to ethanol to make it unsuitable for drinking (see denatured alcohol). It is used in the in vitro synthesis of DNA, ${ }^{4}$ in the synthesis of sulfapyridine (a drug against bacterial and viral infections), antihistaminic drugs tripelennamine and me pyramine, as well as water repellents, bactericides, and herbicides. Some chemical compounds, although not synthesized from pyridine, contain its ring structure. They include B vitamins niacin and pyridoxine, the anti-tuberculosis drug isoniazid, nicotine and other nitrogen-containing plant products. ${ }^{5}$ Historically, pyridine was produced from coal tar and as a byproduct of coal gasification. However, increased demand for pyridine resulted in the development of more economical methods of synthesis from acetaldehyde and ammonia, and more than 20,000 tonnes per year are manufactured worldwide.

A Schiff base (named after Hugo Schiff) is a compound with the general structure $\mathrm{R}_{2} \mathrm{C}=\mathrm{NR}^{\prime}\left(\mathrm{R}^{\prime} \neq\right.$ H). ${ }^{6,7}$ They can be considered a sub-class of imines, being either secondary ketimines or secondary aldimines depending on their structure. The term is often synonymous with azomethine which refers specifically to secondary aldimines (i.e. $\mathrm{R}-\mathrm{CH}=\mathrm{NR}^{\prime}$ where $\left.\mathrm{R}^{\prime} \neq \mathrm{H}\right){ }^{8}$

A number of special naming systems exist for these compounds. For instance a Schiff base derived from an aniline, where $\mathrm{R}^{3}$ is a phenyl or a substituted phenyl, can be called an anil, ${ }^{9}$ while bis-compounds are often referred to as salen-type compounds.

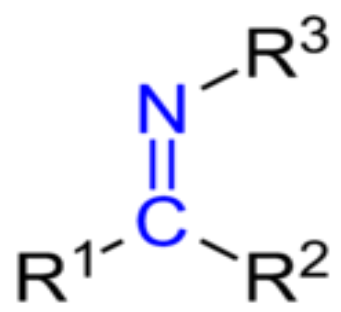


The term Schiff base is normally applied to these compounds when they are being used as ligands to form coordination complexes with metal ions. Such complexes do occur naturally, for instance in Corrin, but the majority of Schiff bases are artificial and are used to form many important catalysts, such as Jacobsen's catalyst.

The $\beta$-lactams are 4-membered cyclic amides derived from 3-aminopropanoic acids. Though the first member synthesized by Staudinger ${ }^{10}$ in 1907 , the $\beta$ lactams as a class acquired importance since the discovery of penicillin which contains $\beta$-lactam unit as an essential structural feature of its molecule, this interest continued unabated because of the therapeutic importance of $\beta$-lactam antibiotics and recent finding of new naturally occurring $\beta$-lactams. As a result of vigorous research, a vast literature has been accumulated over the years, and the chemistry of azetidinones continues to be blossoming field.

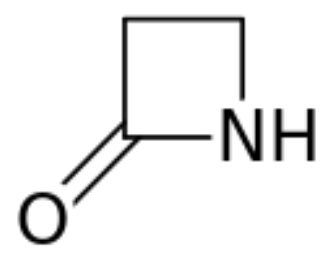

Azetidine and their derivatives have been extensively explored for their applications in the field of medicine205-209. Likewise, azetidin-2-ones are of great importance because of $\beta$-lactam derivatives as an antibacterial agent. ${ }^{11}$ Recently, incorporation of these compounds have witnessed a great upsurge in the treatment of tuberculosis and other chemotherapeutic diseases. Sharma et al 216 reported synthesis and antibacterial activity of some Nsulphonamoylphenylamino-3-chloro-4- phenylazetidin2-ones. Most of the compounds exhibited significant antibacterial activity.Comp. 1 [4(5,6 dimethoxy pyrimidino sulphonamoyl)phenylamino]-3-chloro- 4phenylazetidin-2-one ${ }^{11}$ has been found to be very potent compound against E. coli.
Tuberculosis: Tuberculosis (TB) is an infectious disease usually caused by the bacterium Mycobacterium tuberculosis (MTB). Tuberculosis generally affects the lungs, but can also affect other parts of the body. Most infections do not have symptoms, in which case it is known as latent tuberculosis. ${ }^{12}$ About $10 \%$ of latent infections progress to active disease which, if left untreated, kills about half of those infected. ${ }^{12}$ The classic symptoms of active TB are a chronic cough with blood-

containing sputum, fever, night sweats, and weight loss. ${ }^{12}$ The historical term "consumption" came about due to the weight loss. ${ }^{13}$ Infection of other organs can cause a wide range of symptoms. ${ }^{14}$

Tuberculosis is spread through the air when people who have active TB in their lungs cough, spit, speak, or sneeze ${ }^{[6]}$ People with latent TB do not spread the disease. Active infection occurs more often in people with HIV/AIDS and in those who smoke. ${ }^{12}$ Diagnosis of active TB is based on chest X-rays, as well as microscopic examination and culture of body fluids. Diagnosis of latent TB relies on the tuberculin skin test (TST) or blood tests. ${ }^{15}$

\section{Materials and Method}

All the chemicals are purchased from SD Chemicals. Melting point is determined by using digital melting point(model). Using open capillary tube method.IR spectroscopy is determined by using shimadzu (ftir). Proton NMR is detrmined by using BRUKER.

\section{Synthetic scheme}

Step 1: Synthesis of Schiff base by Grind stone method: Take equimolar Pyridine-4-carbaldehyde $(0.01 \mathrm{~mol})$ and Substituted aromatic amines $(0.01 \mathrm{~mol})$ in to a mortar, to this add2-3 drops of Glacial acetic acid and $5 \mathrm{ml}$ water.The reaction mixture was grounded for 15-30 min. The reaction monitored by TLC .After completion of reaction add $25 \mathrm{ml}$ of water and stirred product for 5min. Seperated out solid was filtered, Washed with water and recrystallized from ethanol to give the corresponding Schiff bases.

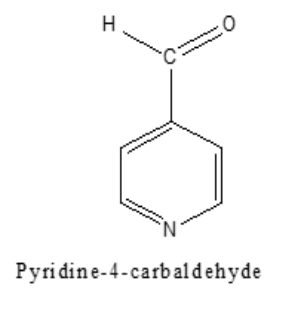

I

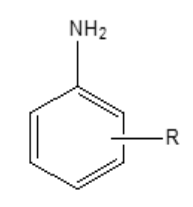

Substituted amines

II

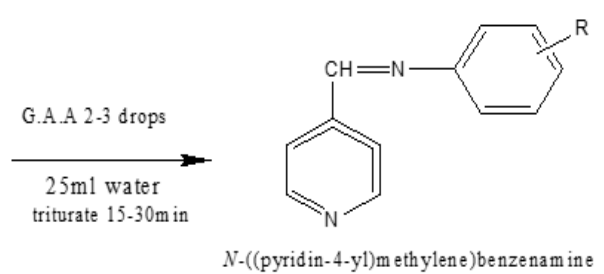

III 
Step 2: Synthesis of pyridine containing Azetidinone derivaties:

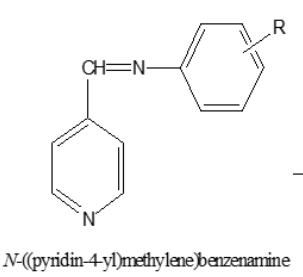

III

Schiff base

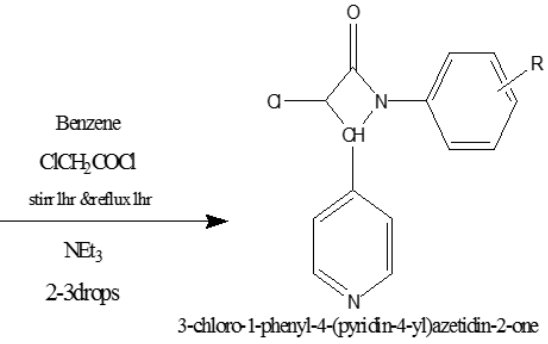

IV

To the Schiff base compounds $(0.01 \mathrm{~mol})$ in absolute benzene $(100 \mathrm{ml})$,add triethylamine (2-3 drops) and chloroacetylchloride $(0.02 \mathrm{~mol})$ were added drop by drop, stirr the mixture for $1 \mathrm{hr}$, further the reaction mixture refluxed for 1 hour .the reaction was monitored by TLC, The reaction mixture was cooled and poured into ice. The solid thus obtained was filtered and recrystallized from ethanol.

\section{Results and Discussion}

Microplate Alamar Blue Assay: The antimycobacterial activity of compounds were assessed against M. tuberculosis using microplate Alamar Blue assay (MABA).This methodology is non-toxic, uses a thermally stable reagent and shows good correlation with propotional and BACTEC radiometric method. Briefly, 200 $\mu 1$ of sterile deionzed water was added to all outer perimeter wells of sterile 96 wells plate to minimized evaporation of medium in the test wells during incubation. The 96 wells plate received $100 \mu \mathrm{l}$ of the Middlebrook 7H9 broth and serial dilution of compounds were made directly on plate. The final drug concentrations tested were 100 to $0.2 \mu \mathrm{g} / \mathrm{ml}$. Plates were covered and sealed with parafilm and incubated at $37^{\circ} \mathrm{C}$ for five days.After this time, $25 \mu$ of freshly prepared 1:1 mixture of Almar Blue reagent and 10\% tween 80 was added to the plate and incubated for 24 hrs. Finally the readings were noted based on the visual colour change. Pink colour in the well indicates growth of the bacteria and blue colour indicates no bacterial growth.

The MIC was defined as lowest drug concentration which prevented the color change from blue to pink.

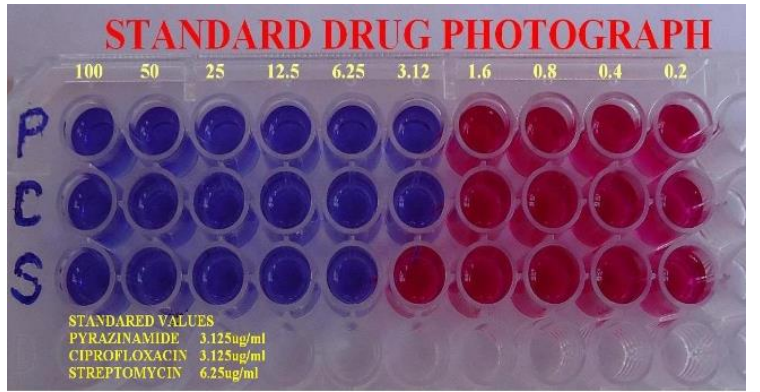

Fig. 1: Anti TB Standard drug photography

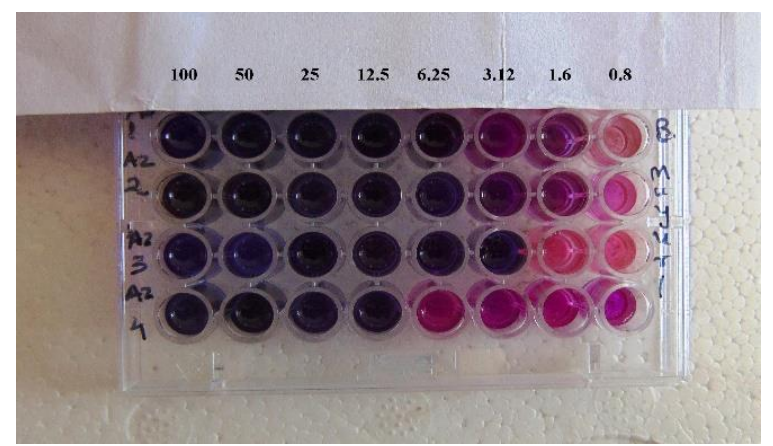

Figure 2: Microplate wells with samples

The minimum inhibitory concentrations of the synthesized derivatives against Mycobacterium tuberculosis obtained through Microplate Alamar Blue Assay method were represented below:

\section{Graph 1: M. tuberculosis MIC values obtained for the synthesized derivatives through MABA}

$$
\operatorname{MIC}(\mu \mathrm{g} / \mathrm{ml})
$$

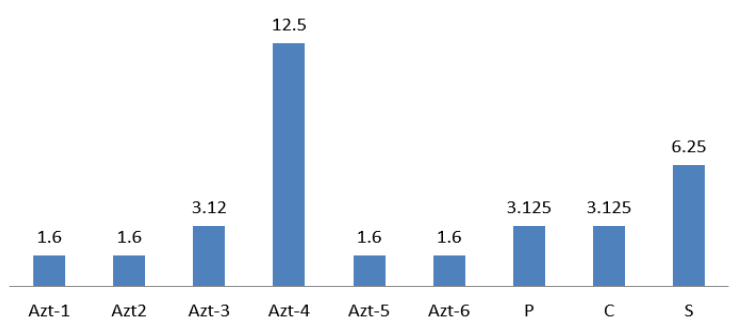

$\mathrm{P}=$ Pyrazinamide, $\mathrm{C}=$ Ciprofloxacine, $\mathrm{S}=$ Streptomycine . 


\section{Discussion}

In the present research work, based on the wide literature survey, novel derivatives of pyridine containing azetidinone derivatives were synthesized in simple two-step facile procedure. All the reactions were monitored by TLC and purification was done by recrystallization technique. All the derivatives were characterized using spectral studies like FT-IR spectroscopy, ${ }^{1} \mathrm{H}-\mathrm{NMR}$ spectroscopy and Mass spectrometry.

All the six derivatives were screened for their invitro anti-tubercular activity using Microplate Alamar Blue Assay method.

In-vitro anti tubercular activity: The anti-tubercular activity of the synthesized derivatives, Azt1-6 was carried out using various concentrations from 100 to 0.8 $\mu \mathrm{g} / \mathrm{ml}(100,50,25,12.5,6.25,3.12,1.6$, and 0.8 $\mu \mathrm{g} / \mathrm{ml})$. Out of all six derivatives Azt1, Azt2 and Azt5 shown more potent activity than standards. Azt3, Azt4 shown equipotent activity when compared to standard. Good to excellent activities with similar minimum inhibitory concentrations compared to that of the standard drugs pyrazinamide, streptomycin, and ciprofloxacin.

Acknowledgement: The authors are thankful to Shri.C.Gangi Reddy garu through Principal of annamacharya college of pharmacy rajampet for provoding necessary facilities to carry out this research work successfully.

\section{References}

1. Sigma-Aldrich. Retrieved 28 November 2011.

2. $\quad$ "Pyridine". Encyclopadia Britannica Online.

3. Mootz, D. (1981). "Crystal structures of pyridine and pyridine trihydrate". The Journal of Chemical Physics. 75 (3):

1517. Bibcode:1981JChPh..75.1517M. doi:10.1063/1.442 204.

4. "Iodine Solution ( $0.02 \mathrm{M}$ in $\mathrm{THF} /$ pyridine $/ \mathrm{H}_{2} \mathrm{O}$ 70:20:10)". Sigma-Aldrich. Retrieved 28 November 2011.

5. "Pyridine". Encyclopædia Britannica Online.

6. IUPAC, Compendium of Chemical Terminology, 2nd ed. (the "Gold Book") (1997). Online corrected version: (2006-) "Schiff base".

7. Schiff, Hugo (1866). "Eine neue Reihe organischer Diamine. Zweite Abtheilung" [A new series of organic diamines. Second part.]. Annalen der Chemie und Pharmacie (in German). 140:92-137.

8. IUPAC, Compendium of Chemical Terminology, 2nd ed. (the "Gold Book") (1997). Online corrected version: (2006-) "azomethines".

9. IUPAC, Compendium of Chemical Terminology, 2nd ed. (the "Gold Book") (1997). Online corrected version: (2006-) "anils".

10. Gilchrist, T. (1987). Heterocyclic Chemistry. Harlow: Longman Scientific. ISBN 0-582-01421-2.

11. Brandt, C.; et al. (2017). "In silico serine $\beta$-lactamases analysis reveals a huge potential resistome in environmental and pathogenic species". SciRep. 7 (43232). Bibcode:2017NatSR...74323 2B. doi:10.1038/srep43232.

12. "Tuberculosis Fact sheet $\mathrm{N}^{\circ} 104 "$ ". WHO. October 2015. Archived from the original on 23 August 2012. Retrieved 11 February 2016.

13. The Chambers Dictionary. New Delhi: Allied Chambers India Ltd. 1998. p. 352. ISBN 978-81-86062-258. Archivedfrom the original on 6 September 2015.

14. Dolin, [edited by] Gerald L. Mandell, John E. Bennett, Raphael (2010). Mandell, Douglas, and Bennett's principles and practice of infectious diseases (7th ed.). Philadelphia, PA: Churchill Livingstone/Elsevier. pp. Chapter 250. ISBN 978-0-443-06839-3

15. Konstantinos A (2010). "Testing for tuberculosis". Australian Prescriber. 33 (1): 12-18. Archived from the original on 4 August 2010. 